\title{
Efficacy of aflibercept with FOLFOX and maintenance with fluoropyrimidine as first-line therapy for metastatic colorectal cancer: GERCOR VELVET phase II study
}

\author{
BENOIST CHIBAUDEL ${ }^{1,2}$, JEAN-BAPTISTE BACHET ${ }^{3}$, THIERRY ANDRÉ ${ }^{4}$, DOMINIQUE AUBY $^{5}$, \\ JÉRÔME DESRAMÉ ${ }^{6}$, GAËL DEPLANQUE ${ }^{7}$, CÉDRIC LECAILLE ${ }^{8}$, CHRISTOPHE LOUVET $^{9}$, \\ CHRISTOPHE TOURNIGAND ${ }^{10}$, VALÉRIE LEBRUN-LY ${ }^{11}$, JÉRÔME DAUBA ${ }^{5}$, GÉRARD LLEDO ${ }^{6}$, \\ MARIE-LINE GARCIA ${ }^{2,4}$, OLIVIER DUBREUIL ${ }^{12}$, NABIL BABA HAMED ${ }^{7}$, \\ AURÉLIA MEURISSE ${ }^{13}$, ANNETTE K. LARSEN ${ }^{14}$, ANNEMILAÏ TIJERAS-RABALLAND ${ }^{15}$, \\ FRANCK BONNETAIN $^{13}$ and AIMERY DE GRAMONT ${ }^{1,2}$
}

\footnotetext{
${ }^{1}$ Department of Medical Oncology, Franco-British Institute, 92300 Levallois-Perret; ${ }^{2}$ Centre de Recherche Saint-Antoine, INSERM U938, Hospital Saint-Antoine, 75012 Paris; ${ }^{3}$ Department of Gastroenterology, Sorbonne University,

Pierre and Marie Curie University Paris 6, CH Universitaire Pitié-Salpétrière, 75013 Paris;

${ }^{4}$ Department of Medical Oncology, Sorbonne University, Hospital Saint-Antoine, 75012 Paris;

${ }^{5}$ Department of Medical Oncology, CH de Mont-de-Marsan, Hospital Layné, 40024 Mont-de-Marsan;

${ }^{6}$ Department of Gastroenterology, Private Hospital Jean Mermoz, 69008 Lyon; ${ }^{7}$ Department of Medical Oncology,

Hospital Saint-Joseph, 75014 Paris; ${ }^{8}$ Department of Medical Oncology, Polyclinic Bordeaux Nord,

33300 Bordeaux; ${ }^{9}$ Department of Medical Oncology, Institut Mutualiste Montsouris, 75014 Paris;

${ }^{10}$ Department of Medical Oncology, Henri Mondor Hospital, 94010 Créteil; ${ }^{11} \mathrm{CHU}$ Dupuytren,

87042 Limoges; ${ }^{12}$ Department of Gastroenterology, Hospital Pitié-Salpétrière, 75013 Paris;

${ }^{13}$ Department of Biostatistics, CHU Besançon, 25030 Besançon; ${ }^{14}$ Cancer Biology and Therapeutics,

Centre de Recherche Saint-Antoine, INSERM U938, Sorbonne Université Faculté de Médecine,

75012 Paris; ${ }^{15}$ Preclinical and Translational Laboratory, AFR Oncology, Paris, France
}

Received May 24, 2018; Accepted December 18, 2018

DOI: 10.3892/ijo.2019.4709

\begin{abstract}
Aflibercept in combination with 5-fluorouracil (5-FU)/irinotecan improves overall survival in the second-line therapy of patients with metastatic colorectal cancer (mCRC). In this study, we evaluated the effects of aflibercept in first-line therapy with FOLFOX followed by maintenance with fluoropyrimidine. VELVET was a prospective, single-arm multicenter phase II study (completed). Patients with previously untreated, unresectable, evaluable or measurable mCRC, with an age $\geq 18$ years, and an ECOG performance status of $0-2$ received 6 cycles of modified FOLFOX7 (5-FU/folinic acid and oxaliplatin) with aflibercept at $4 \mathrm{mg} / \mathrm{kg}$ every 2 weeks followed by maintenance therapy with fluoropyrimidine with
\end{abstract}

Correspondence to: Dr Benoist Chibaudel, Department of Medical Oncology,Franco-British Institute, 4 rue Kléber, 92300 Levallois-Perret, France

E-mail: benoist.chibaudel@ihfb.org

Key words: colorectal cancer, chemotherapy, angiogenesis, FOLFOX, aflibercept aflibercept until disease progression or limiting toxicity. The reintroduction of oxaliplatin was performed at first progression. The primary endpoint was progression-free survival (PFS) at 6 months. From May, 2013 to May, 2014, 49 patients were included and 48 were evaluable for response. In total, 33 patients $(67.4 \%)$ were alive without progression at 6 months. The Kaplan-Meier survival 6-month and 1-year PFS rates were 79.1 and $36.1 \%$, respectively, and the median PFS was 9.3 months (95\% CI, 8.3-12.5). The objective response rate was $59.2 \%(\mathrm{~N}=29 / 49)$. The most common $(\geq 10 \%)$ grade $3-4$ adverse events were hypertension $(23 \%)$, fatigue $(15 \%)$, neutropenia $(12 \%)$, neuropathy $(12 \%)$ and stomatitis $(10 \%)$. Three $(6 \%)$ treatment-related deaths occurred: One from stroke, one from pulmonary embolism and one from neutropenic sepsis. On the whole, this study demonstrates the efficacy of aflibercept in combination with an oxaliplatin-based regimen in the first-line therapy of patients with mCRC. A strict monitoring of blood pressure and immediate management of hypertension during therapy is mandatory.

\section{Introduction}

Colorectal cancer (CRC) is the third most common type of cancer in western countries and the third most common cause of 
cancer-related mortality (1). The median overall survival (OS) of patients with previously untreated with unresectable advanced CRC ranges from 25 to 30 months, when combining molecular targeted therapies and chemotherapy (2).

Standard first-line therapy is doublet or triplet-chemotherapy combined with targeting agents, including either the monoclonal antibody, bevacizumab, that inhibits angiogenesis through vascular endothelial growth factor (VEGF)-A or the monoclonal antibodies, cetuximab and panitumumab, which inhibit the epidermal growth factor receptor (EGFR) pathway (3-7); the latter option is restricted to approximately half the patients harboring wild-type $R A S$ in their tumor (8). Oxaliplatin combined with 5-FU (FOLFOX) is one of most commonly used first-line treatment combinations (9). This regimen is optimized with the oxaliplatin stop-and-go strategy (OPTIMOX), which consists of 6 cycles as induction therapy followed by maintenance with fluoropyrimidine without oxaliplatin and later, at progression, reintroduction of the full regimen. Maintenance therapy reduces the frequency and severity of the cumulative neuropathy observed with oxaliplatin (10). Bevacizumab with fluoropyrimidine is considered as a standard for maintenance therapy (11).

Aflibercept is a recombinant fusion protein consisting of the extracellular domains VEGFR1 and VEGFR2 fused to the Fc portion of human immunoglobulin G1. Aflibercept binds VEGF-A and VEGF-B with high affinity $(\mathrm{Kd}<1 \mathrm{pM})$ and placental growth factor (PIGF) with lower affinity $(\mathrm{Kd} 39 \mathrm{pM})$, leading to the blockade of tumor angiogenesis and vascular permeability. The combination of aflibercept to the standard FOLFIRI regimen in patients with metastatic CRC (mCRC) has been shown to improve OS [primary endpoint, 12.1-13.5 months; hazard ratio (HR), 0.82; $\mathrm{P}=0.003$ ], progression-free survival (PFS, 4.7-6.9 months; HR, 0.76; $\mathrm{P}<0.001)$, and the objective response rate (ORR, 11.1-19.8\%; $\mathrm{P}<0.001)(12)$. This effect was observed whether or not patients had received prior bevacizumab therapy.

The aim of this study was to evaluate the efficacy and safety of the aflibercept and an oxaliplatin-based chemotherapeutic regimen combination in first-line therapy in order to determine whether aflibercept has the potential to challenge bevacizumab in the first-line treatment of mCRC.

\section{Patients and methods}

Study population. The main patient inclusion criteria were as follows: an age $\geq 18$ years, an Eastern Cooperative Oncology Group performance status (ECOG PS) of 0 to 2, histologically or cytologically confirmed unresectable $\mathrm{mCRC}$ and no prior treatment for metastatic disease.

Study design and treatment schedule. This was a prospective, single-arm, multicenter phase II study. All patients provided written inform consent before enrollment. The study was carried out in accordance with the declaration of Helsinki and Good Clinical Practice guidelines. This study was approved by the Ethics Committee (CPP Ile de France VI Groupe Hospitalier Pitié Salpêtrière PARIS) of our institution.

Patients received intravenously modified FOLFOX7 with aflibercept as induction therapy every 2 weeks for 6 cycles as follows: Aflibercept $4 \mathrm{mg} / \mathrm{kg}$, oxaliplatin $100 \mathrm{mg} / \mathrm{m}^{2}$, folinic acid $400 \mathrm{mg} / \mathrm{m}^{2}$ and 5 -FU $3,000 \mathrm{mg} / \mathrm{m}^{2}$. In patients without progression or non-amenable to surgery, induction therapy was followed by maintenance therapy with aflibercept and fluoropyrimidine (either 5-FU or capecitabine) until disease progression or limiting toxicity. Dose postponements or reductions were permitted to manage treatment-related adverse events.

Endpoints. The primary endpoint was PFS, defined as the time from the date of inclusion to the date of progression or death (from any cause). Patients alive without documented objective progressive disease $(\mathrm{PD})$ at the time of the final analysis were censored at the date of their final objective tumor assessment. OS was defined as the time from the date of inclusion to the date of patient death (from any cause) or to the last date the patient was known to be alive. Patients still alive at the time of the analysis were censored using the date of final news. The duration of disease control (DDC) was defined as the sum of PFS of each active treatment course (13).

The ORR was defined as the proportion of patients having either complete response (CR) or partial response (PR) according to RECIST version 1.1 (14). The optimal ORR was defined as the optimal response recorded from the beginning of treatment until treatment failure, taking as reference for PD the smallest measurements recorded since the beginning of treatment. The early response rate was evaluated at the first disease evaluation (i.e., 2 months). The disease control rate (DCR) was defined as the percentage of patients who achieved CR, PR, or stable disease (SD).

The reintroduction rate was defined as the number of patients who received reintroduction of oxaliplatin after disease progression during aflibercept-based maintenance therapy. The absolute reintroduction rate was calculated for all included patients and the relative reintroduction rate was calculated for patients eligible to reintroduction, excluding patients having progressed during induction therapy, amenable to surgery or having a residual sensory neuropathy grade $>1$. The curative surgery rate was assessed globally and per sequence of therapy.

Toxicity was evaluated according to the US National Cancer Institute's Common Terminology Criteria for Adverse Events (NCI CTCAE) version 4.03 (15). Health-related quality of life (HRQoL) assessments were performed at baseline, and every 2 months thereafter, using the Quality of Life Questionnaire Core 30 (QLQ-C30) (French version) (16). The survival prognosis was assessed through the GERCOR prognostic model (17), using two-baseline (pre-treatment) parameters: ECOG PS and serum lactate dehydrogenase levels.

Sample size. According to Simon's Minimax two-stage design (18) with a two-sided 5\% type I error, a power of $80 \%$, and a $15 \%$ improvement in PFS rate at 6 -month from $70 \%$ ( $\mathrm{H0}$, considered as uninteresting to pursue any further investigation) to $85 \%$ ( $\mathrm{H} 1$, considered as promising to warrant further investigation), it was required that we enroll 49 patients, including a 5\% drop-out. If $>16$ patients were free of progression or death at 6 months from inclusion among the first 23 evaluable patients (stage 1), the trial could be pursued to the second stage with further 26 patients. If at least 40 patients were free of progression or death among the 


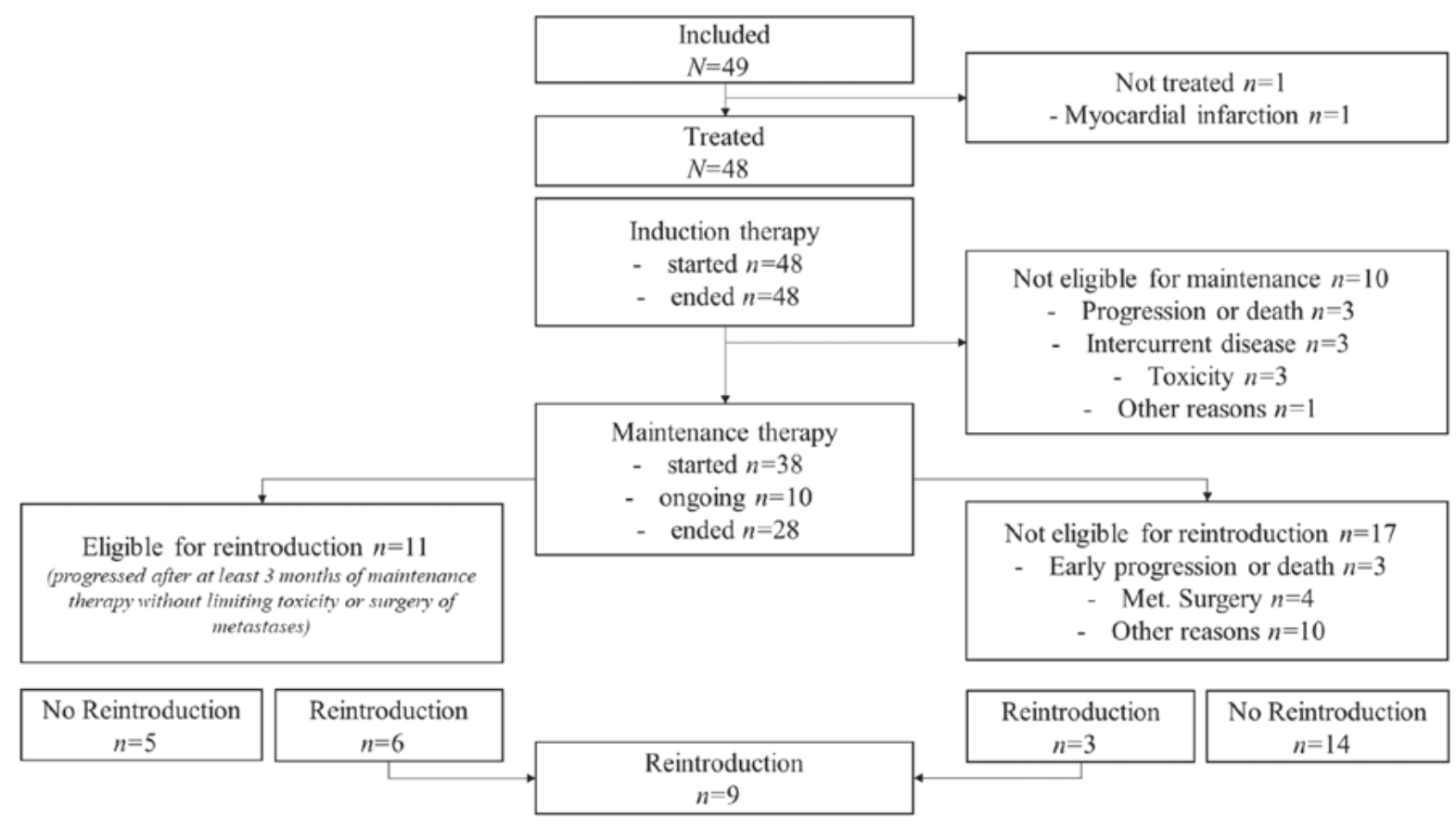

Figure 1. Study flow diagram.

49 included patients (stage 2), treatment could be considered as promising for further evaluation.

Statistical analysis. The primary analysis of efficacy used the intent-to-treat (ITT) population, i.e., including all recruited patients regardless of their eligibility. The confirmative analysis was conducted in the ITT population of eligible patients and in the per-protocol (PP) population comprising all patients who have received at least 2 cycles of the allocated treatment and without any major protocol deviations. The safety analysis included all patients who received at least one dose of any study drug. Follow-up and survival were estimated using the reverse Kaplan-Meier method (19) and Kaplan-Meier method (20), respectively, and were described using median with $95 \%$ confidence interval (CI). A linear mixed effects model (repeated measures of variance) was used as to analyze longitudinal changes of HRQoL at baseline, and every 2 months. All patients who completed at least one baseline HRQoL assessment were included. Qualitative variables were described using percentage and means (SD), and continuous variables using medians (minimum-maximum). Fisher's exact test was used for comparison of proportions. The log-rank test was used to compare survival curves, and Cox proportional-hazards regression was used to analyze the effect of several risk factors on survival. The cut-off date for statistical analysis was December, 2015.

Circulating biomarkers. The plasma concentration of 31 biomarkers (3 panels), including cytokines, growth factors, or soluble receptors was determined using multiplexing immunoassays on a Biorad ${ }^{\circledR}$ Bioplex platform. PlGF and neuropillin 1 levels were determined by enzyme-linked immunosorbent assays (ELISA; R\&D Systems, Minneapolis, MN,USA). The samples and standards were prepared in duplicate according to the manufacturer's protocol. Plates were incubated for $2 \mathrm{~h}$, washed 4 times, and incubated with enzyme-conjugated antibodies for an additional $2 \mathrm{~h}$ at room temperature. The wells were then washed 4 times and substrate was added for $20 \mathrm{~min}$ also at room temperature, in the dark. Finally, stop solution was added to each well, and the absorptions at $450 \mathrm{~nm}$ were determined using a luminometer plate reader. Plasma markers were evaluated at baseline, and before each induction therapy infusion, for a total of 7 time points.

\section{Results}

Study conduct. From May, 2013 to May, 2014, 49 patients were included in 9 French centers (Fig. 1). In total, 23 (46.9\%) and $26(53.1 \%)$ patients were included in the Simon's stage 1 and stage 2 , respectively.

Patient characteristics. The patient and tumor baseline characteristics are presented in Table I. The median age was 62.9 years, ranging from 32 to 86 years. In total, $20(40.8 \%)$ patients were 70 years or older, $19(38.8 \%)$ had a medical history of hypertension, and $18(36.7 \%)$ had liver-limited metastatic disease. According to the GERCOR prognostic model, $13(26.5 \%)$ patients were at high-risk for death at study entry.

Treatment administration. One patient did not receive study treatment due to myocardial infarction.

Induction therapy. A total of $48(97.9 \%)$ patients received at least one treatment dose, and $46(93.8 \%)$ received at least 2 cycles of the full therapy. A total of 268 cycles of induction therapy were administered with a mean number of 5.6 cycles per patient. In total, 19/268 (7.1\%) cycles were postponed.

Maintenance therapy. Following induction therapy, $10(20.8 \%)$ patients did not receive the planned maintenance therapy with fluoropyrimidine and aflibercept due to limiting toxicity $(n=4)$, progression or death $(n=3)$, or interrupted 
Table I. Patient and tumor baseline characteristics.

\begin{tabular}{lc}
\hline Baseline characteristics & $\begin{array}{c}\text { No. of patients } \\
(\mathrm{n}=49)\end{array}$ \\
\hline
\end{tabular}

\section{Sex}

Male

Female

Age, years

$<70$

$\geq 70$

ECOG performance status

0

1

2

Number of metastatic organ sites

1

$\geq 2$

Metastatic disease

Liver

Lung

Node

Peritoneal

Primary tumor sidedness

Right

Left

Initial disease stage

I-III (metachronous)

IV (synchronous)

Prior primary tumor resection

Yes

No

26

23

Prior adjuvant chemotherapy

Yes

No

$R A S$ mutational status

Wild-type

Mutated

Unknown

White blood cell count

$<10,000 / \mathrm{mm}^{3}$

$\geq 10,000 / \mathrm{mm}^{3}$

Platelet count

$\leq 1 \times$ ULN

$>1 \times$ ULN

Lactate dehydrogenase level

$\leq 1 \times$ ULN

$>1 \times$ ULN

Missing data

Alkaline phosphatase level

$\leq 1 \mathrm{xULN}$

$>1 \mathrm{xULN}$

Carcinoembryonic antigen level

$\leq 1 \times$ ULN

$>1 \times$ ULN

Missing data
30

19

23

22

4

26

23

37

16

15

8

20

29

6

43

20

29

18

27

4

38

11

39

10

53.1

46.9

61.2

38.8

46.9

44.9

8.2

53.1

46.9

75.5

32.6

30.6

16.3

40.8

59.2

12.2

87.8

40.8

59.2

10.2

89.8

36.7

55.1

8.2

77.5

22.5

79.6

20.4

38.8

53.1

8.2

63.3

36.7

10

20.4

57.1

2.0
Table I. Continued.

\begin{tabular}{lcr}
\hline Baseline characteristics & $\begin{array}{c}\text { No. of patients } \\
(\mathrm{n}=49)\end{array}$ & $\%$ \\
\hline GERCOR prognostic score & & \\
Low-risk & 8 & 16.3 \\
Intermediate-risk & 24 & 49.0 \\
High-risk & 13 & 26.5 \\
Missing data & 4 & 8.2 \\
\hline
\end{tabular}

ECOG, Eastern Cooperative Oncology Group; ULN, upper limit of normal.

administration of aflibercept for $>21$ days $(n=2)$, or investigator decision $(n=1)$. Among the $38(79.2 \%)$ patients who received maintenance therapy (fluorouracile-based, $n=37$; capecitabine-based, $n=1), 10(26.3 \%)$ patients were still on maintenance therapy. A total of 415 cycles of maintenance therapy were administered, with a mean number of 10.9 cycles per patient. In total, $48 / 415$ (11.6\%) cycles were postponed. The median duration of maintenance therapy was 5.5 months (95\% CI, 3.7-9.9).

Reintroduction. At the time of analysis, 11 patients were eligible for oxaliplatin reintroduction and 6 patients received an oxaliplatin reintroduction. Three other patients had an unplanned reintroduction of FOLFOX-aflibercept after surgery of metastasis $(n=2)$ or an early progression $(n=1)$.

\section{Efficacy}

Progression-free survival. At Simon's stage $1 \quad(\mathrm{n}=23)$, 17 (73.9\%; 95\% CI, 56.0-91.9) patients were alive without disease progression at 6 months. In the ITT population $(n=49)$, 33 (67.4\%; 95\% CI, 54.2-80.5) patients were alive without disease progression at 6 months, $12(24.5 \%)$ patients were considered as failure (5 patients had RECIST progression, 4 patients had clinical progression, and 3 patients died), and $4(8.2 \%)$ patients were not evaluated for other reasons (no tumor measure, patient decision, surgery of the primary tumor and investigator's decision). Following a median follow-up of 22.5 months (95\% CI, 20.9-24.5), the median PFS was 9.3 months (95\% CI, 8.3-12.5). The 6-month and 1-year PFS rates were 79.1 and $36.1 \%$, respectively. The median PFS from the beginning of maintenance therapy $(n=38)$ was 7.4 months (95\% CI, 5.9-9.5). Patients with prior hypertension or high systolic blood pressure $(\geq 140 \mathrm{mmHg})$ at study entry had a significantly shorter PFS (HR, 2.37 and 2.61, respectively) than the other subgroups (Table II).

Overall survival. At the time of analysis, 26 (53.1\%) patients were alive. The median follow-up was 10.9 months (95\% CI, 9.9-12.0). The median OS was 22.2 months (95\% CI, 18.2-24.7). The 6-month and 1-year survival rates were 91.8 and $79.6 \%$, respectively.

Tumor response. A total of 45/49 (91.8\%) patients were evaluated, and $4(8.2 \%)$ patients were not evaluable for tumor response (2 patients with early death, 1 with gastrointestinal perforation, and 1 patient was not treated). The ORR (CR or PR) was observed in $29(59.2 \%)$ of the 49 patients in the ITT population, and in $28(60.9 \%)$ of the 46 patients in the PP population (Table III). 
Table II. Progression-free survival in the ITT population.

\begin{tabular}{lllllll}
\hline Parameter & No. Events & Median (months) & $95 \%$ CI & Hazard ratio & $95 \%$ CI $\quad$ P-value
\end{tabular}

All patients
Age (years)
$<65$
$\geq 65$
Tumor response

CR or PR

SD or PD

$\begin{array}{rr}49 & 23 \\ 25 & 9 \\ 24 & 14\end{array}$

23

$29 \quad 13$

$20 \quad 10$

$29 \quad 12$

$20 \quad 11$

$\begin{array}{rr}23 & 9.5 \\ 9 & 11.9 \\ 4 & 8.8\end{array}$

8.7

Body mass index $\left(\mathrm{kg} / \mathrm{m}^{2}\right)$

$<25$

$\geq 25$

$34 \quad 13$

$<140$

$\geq 140$

$13 \quad 8$

9.9
9.5

9.5

5.0-11.0

$$
9.5
$$

8.7-11.9

7.0-11.0

9.3-12.6

ref

1.86

$0.82-4.21$

0.136

Diastolic blood pressure $(\mathrm{mmHg})$

$<90$
$\geq 90$

$40 \quad 18$

12.6

8.7

8.7-12.6

5.7-11.0

ref

1.71

$0.71-4.15$

0.191

73

9.1

11.0

8.3-12.6

5.7-11.0

ref

1.81

$0.76-4.29$

0.148

Prior hypertension

No

$30 \quad 10$

$19 \quad 13$

11.9

9.3-12.6

8.8

6.8-9.9

ref
2.61

0.87-7.74

0.023

Yes

$\begin{array}{ll}26 & 12 \\ 23 & 11\end{array}$

\section{5}

8.7-12.6

6.4-9.9

ref

0.90

$0.28-2.93$

0.866

umber of metastatic sites

$$
>1
$$

8.8

ref

2.37

$1.00-5.56$

0.033

Liver involvement

No
Yes

$\begin{array}{rr}12 & 3 \\ 37 & 20\end{array}$

$$
-
$$

8.7-12.6

ref
1.36

0.59-3.15

0.455

ECOG PS

0

1-2

$23 \quad 10$

$26 \quad 13$

11.0

9.5

7.6-12.6

8.7-11.9

1.26

0.56-2.86

0.562

Sex

Male

$\begin{array}{ll}23 & 11 \\ 26 & 12\end{array}$

9.1
9.5

$7.7-12.6$
$8.3-11.9$

ref

0.96

$0.42-2.18$

0.922

\begin{tabular}{|c|c|c|c|c|c|c|c|}
\hline Mutated & 25 & 10 & 9.9 & $8.7-11.0$ & ref & & \\
\hline Wild-type & 20 & 11 & 9.5 & $7.7-12.6$ & 1.12 & $0.48-2.65$ & 0.784 \\
\hline \multicolumn{8}{|l|}{ Weight (kg) } \\
\hline$<70$ & 27 & 10 & 9.5 & $8.7-9.5$ & ref & & \\
\hline$\geq 70$ & 22 & 13 & 9.9 & $6.4-11.9$ & 1.41 & $0.62-3.19$ & 0.406 \\
\hline
\end{tabular}

KRAS exon 2

mutation status

'ref' indicates the reference group for comparison. ITT, intent-to-treat; CR, complete response; PR, partial response; SD, stable disease; PD, progressive disease; ECOG, Eastern Cooperative Oncology Group; ULN, upper limit of normal.

Salvage surgery. A total of $6(8.4 \%)$ patients had liver surgery during maintenance therapy for the resection of 2 to 7 lesions per patient with a maximum tumor size of 15 to $55 \mathrm{~mm}$. The percentage of necrosis ranged between 50 and $100 \%$. Of the 4 patients who underwent salvage surgery, 1 patient had a complete pathological response and 1 patient had $<1 \%$ viable residual tumor cells. A R0 resection was achieved in 1 patient and $\mathrm{R} 1$ in 3 patients.

Safety. The most common ( $\geq 10 \%)$ treatment-related grade 3-4 adverse events were hypertension (23\%), fatigue (15\%), neutropenia (12\%), neuropathy (12\%) and stomatitis (10\%; Table IV). 
Table III. Tumor response in the ITT and PP populations.

\begin{tabular}{lcc}
\hline Response & $\begin{array}{c}\text { Intent-to-treat } \\
\text { population } \\
(\mathrm{n}=49), \\
\text { no. }(\%)\end{array}$ & $\begin{array}{c}\text { Per protocol } \\
\text { population } \\
(\mathrm{n}=46), \\
\text { no. }(\%)\end{array}$ \\
\hline Optimal response rate & & \\
Complete response & $2(4.1)$ & $2(4.3)$ \\
Partial response & $27(55.1)$ & $26(56.5)$ \\
Stable disease & $15(30.6)$ & $15(30.4)$ \\
Progressive disease & $1(2.0)$ & 1 \\
Not evaluable & $4(8.2)$ & $2(4.3)$ \\
Objective response rate & $29(59.2)$ & $28(60.9)$ \\
Disease control rate & $44(89.8)$ & $43(93.5)$ \\
\hline
\end{tabular}

ITT, intent-to-treat; PP, per-protocol.

The majority of events occurred during induction therapy and decreased following the termination of oxaliplatin, apart from fatigue and stomatitis. Severe (grade 3 or 4) hypertension occurred in 11 (22.9\%) patients, mainly during induction therapy $(n=10 / 11,90.9 \%)$, and was reversed in most cases before maintenance therapy. In total, 26 (54.2\%) and $22(45.8 \%)$ patients had treatment-related hypertension grade $0-1$ and 2-4, respectively (Table V). Patients with grade 2-4 hypertension were more frequently women $(\mathrm{P}=0.081)$, had more frequently high systolic blood pressure at study entry $(\mathrm{P}=0.001)$, had a higher number of metastatic sites involved $(\mathrm{P}=0.008)$, and had more treatment-induced proteinuria $(\mathrm{P}=0.016)$. There were 3 (6.1\%; 95\% CI, -0.6-12.8) treatment-related deaths due to stroke in the context of hypertension $(n=1)$, pulmonary embolism $(n=1)$ and neutropenic sepsis $(n=1)$.

Health-related quality of life. A total of 47 (95.9\%) patients filled the baseline HRQoL questionnaire. In total, 10 patients with no follow-up measure had a lower baseline HRQoL level than other patients. The median time until definitive deterioration or death varied from 5.6 months (99\% CI, 2.0-10.3) for physical functioning to 8.9 months $(99 \% \mathrm{CI}, 3.9-14.1)$ for emotional functioning. For sensitivity analysis, all medians for targeted dimensions were $<5$ months. An abnormal monocyte level was associated with a shorter time until the definitive deterioration of emotional functioning or death $(\mathrm{HR}=3.7 ; 99 \% \mathrm{CI}, 1.1-12.0)$.

Circulating biomarkers. The exposure to aflibercept with FOLFOX was associated with an increase in the levels of soluble (s)VEGFR1 and PIGF after the first infusion. High baseline levels of sVEGFR2, sEGFR, G-CSF, prolactin and low baseline levels of VEGFA and migration inhibitory factor (MIF) were associated with a higher response rate. High baseline levels of PIGF predict a poor PFS and OS

Table IV. A summary of the adverse events by System Organ Class.

\begin{tabular}{|c|c|c|c|c|c|c|}
\hline \multirow[b]{2}{*}{ NCI CTCAE } & \multicolumn{2}{|c|}{ Whole strategy $(n=48)$} & \multicolumn{2}{|c|}{ Induction $(\mathrm{n}=48)$} & \multicolumn{2}{|c|}{ Maintenance $(n=28)$} \\
\hline & $\begin{array}{c}\text { Any grade } \\
\text { no. }(\%)\end{array}$ & $\begin{array}{c}\text { Grade } 3-4 \\
\text { no. }(\%)\end{array}$ & $\begin{array}{c}\text { Any grade } \\
\text { no. }(\%)\end{array}$ & $\begin{array}{c}\text { Grade } 3-4 \\
\text { no. }(\%)\end{array}$ & $\begin{array}{c}\text { Any grade } \\
\text { no. }(\%)\end{array}$ & $\begin{array}{c}\text { Grade 3-4 } \\
\text { no. }(\%)\end{array}$ \\
\hline Neutrophil count decreased & $18(37)$ & $6(12)$ & $18(37)$ & $5(10)$ & $3(11)$ & $1(4)$ \\
\hline Platelet count decreased & $21(44)$ & $2(4)$ & $19(40)$ & $2(4)$ & $7(25)$ & $0 \quad(0)$ \\
\hline Anemia & $29(60)$ & $1(2)$ & $27(56)$ & $1 \quad(2)$ & $11(39)$ & $0 \quad(0)$ \\
\hline Febrile neutropenia & 1 (6) & $1(6)$ & 1 (6) & $1(6)$ & $0 \quad(0)$ & $0 \quad(0)$ \\
\hline Nausea & $35(73)$ & $0 \quad(0)$ & $32(67)$ & $0 \quad(0)$ & $14(50)$ & $0 \quad(0)$ \\
\hline Vomiting & $20(42)$ & $1(2)$ & $18(37)$ & $1 \quad(2)$ & $2(7)$ & $0 \quad(0)$ \\
\hline Mucositis oral & $35(73)$ & $5(10)$ & $29(60)$ & $2(4)$ & $16(57)$ & $3(11)$ \\
\hline Diarrhea & $27(56)$ & $2(4)$ & $23(48)$ & $2(4)$ & $10(36)$ & $0 \quad(0)$ \\
\hline Peripheral sensory neuropathy & $43(90)$ & $6(12)$ & $43(90)$ & $4(8)$ & $20(71)$ & $2(7)$ \\
\hline Palmar-plantar erythrodysesthesia syndrome & $17(35)$ & $4(8)$ & $11(23)$ & 1 (2) & $13(46)$ & $4(14)$ \\
\hline Alopecia & $11(23)$ & $5(10)^{\mathrm{b}}$ & $7(15)$ & $3(6)^{\mathrm{b}}$ & $7(25)$ & $2(7)^{\mathrm{b}}$ \\
\hline Fatigue & $33(69)$ & $7(15)$ & $30(62)$ & $5(10)$ & $15(31)$ & $3(11)$ \\
\hline Hypertension & $26(54)$ & $11(23)$ & $26(54)$ & $10(21)$ & $14(50)$ & $2(7)$ \\
\hline Venous thromboembolic event & 1 (2) & 1 (2) & 1 (2) & 1 (2) & $0 \quad(0)$ & $0 \quad(0)$ \\
\hline Arterial thromboembolic event & $2(4)$ & $2(4)$ & $2(4)$ & $2(4)$ & $0 \quad(0)$ & $0 \quad(0)$ \\
\hline Proteinuria & $17(35)$ & $3(6)$ & $9(19)$ & $1(2)$ & $11(29)$ & $2(7)$ \\
\hline Gastrointestinal perforation & $2(4)$ & $2(4)$ & $1(2)$ & $1(2)$ & 1 (4) & $1(4)$ \\
\hline Hemorrhage & $9(19)$ & 1 (2) & $5(10)$ & 1 (2) & $5(18)$ & $0 \quad(0)$ \\
\hline Fistula & 1 (2) & $0 \quad(0)$ & $0 \quad(0)$ & $0 \quad(0)$ & $1(4)$ & $0 \quad(0)$ \\
\hline
\end{tabular}

${ }^{a}$ Whole strategy includes induction, maintenance, reintroduction, and maintenance following reintroduction. ${ }^{\mathrm{b}} \mathrm{Alopecia}$ grade 2 . NCI CTCAE, National Cancer Institute Common Terminology Criteria for Adverse Events. 
Table V. Patient baseline characteristics and clinical outcomes according to the occurrence of hypertension during study treatment.

Characteristic

Grade 0-1 hypertension $(n=26)$,

no. $(\%)$

$17(65.4)$

9 (34.6)

$15 \quad(57.7)$

$11 \quad(42.3)$

$18 \quad(69.2)$

8 (30.8)

Yes

$26(100.0)$

$0 \quad(0.0)$

Yes

Prior history of venous TEE

No

$26(100.0)$

Yes

$0 \quad(0.0)$
0

Baseline systolic blood pressure $(\mathrm{mmHg})$

$<120$

$8 \quad(30.8)$

120-139

$15 \quad(57.7)$

140-159

$>160$

Missing

$2 \quad(7.7)$

$\begin{array}{ll}0 & (0.0)\end{array}$

$1 \quad(3.8)$

Baseline diastolic blood pressure $(\mathrm{mmHg})$

$<80$
$80-89$
$90-99$
$\geq 100$
Missing
Weight $(\mathrm{kg})$
$<70$
$\geq 70$
Body mass index $\left(\mathrm{kg} / \mathrm{m}^{2}\right)$
$<25$
$\geq 25$

Number of metastatic sites

1

$>1$

$12(46.2)$

$10(38.5)$

3 (11.5)

$0 \quad(0.0)$

$1 \quad(3.8)$

$16 \quad(61.5)$

$10(38.5)$

$18 \quad(69.2)$

8 (30.8)

$19(73.1)$

7 (26.9)

$7 \quad(26.9)$

$19(73.1)$

$10 \quad(38.5)$

$13 \quad(50.0)$

3 (11.5)

Unknown

Time to metastasis

Metachronous

3 (11.5)

Synchronous

$23(88.5)$

$11 \quad(42.3)$

$13(50.0)$

2 (7.7)
Grade 2-4 hypertension $(\mathrm{n}=22), \quad \mathrm{P}$-value no. $(\%)$

$\begin{array}{rrr}8 & (36.4) & 0.081 \\ 14 & (63.6) & \\ 14 & (63.6) & 0.771 \\ 8 & (36.4) & \end{array}$

12 (54.5)

0.375

$10 \quad(45.5)$

$20(90.0)$

0.205

2 (9.1)

$22(100.0)$

1.000

$0 \quad(0.0)$
0

3 (13.6)

$0.001^{\mathrm{a}}$

7 (31.8)

9 (40.9)

2 (9.1)

1 (4.5)

$13(59.1)$

$0.686^{\mathrm{a}}$

4 (18.2)

2 (9.1)

2 (9.1)

1 (4.5)

$11(50.0)$

0.561

$11(50.0)$

$11(50.0)$

0.239

$11(50.0)$

7 (31.8)

0.008

15 (68.2)

$5 \quad(22.7)$

1.000

17 (77.3)

9 (40.9)

1.000

12 (54.5)

$1 \quad$ (4.5)

3 (13.6)

19 (86.4)

1.000

$11 \quad(50.0)$

$9 \quad(40.0)$

2 (9.1) 
Table V. Continued.

\section{Characteristic} Grade 0-1 hypertension $(n=26)$, no. $(\%)$

$16(61.5)$

$10(38.5)$

$25(96.2)$

1 (3.8)

$14(53.8)$

$12(46.2)$

$<90$

$15(57.7)$

$11(42.3)$

$20(76.9)$

$6(23.1)$

$10(38.5)$

$13(50.0)$

1 (3.8)

Missing

Carcinoembryonic antigen level

$$
\leq 1 \times \text { ULN }
$$$$
6(23.1)
$$

$>1 \times$ ULN

$20(76.9)$

$11(36.7)$

19 (63.3)

High

Treatment outcomes, efficacy

Tumor response (CR or PR)

No

$12(46.2)$

14 (53.8)

Treatment outcomes, safety

\section{Arterial TEE}

No

$25(96.2)$

1 (3.8)

Hemorrhage

No

$23(88.5)$

$3(11.5)$

Proteinuria

No

$21(80.8)$

5 (19.2)

On treatment death

No

$24(92.3)$

$2(7.7)$

Serious adverse events reported

No

$12(46.2)$

8 (30.8)

$6(23.1)$

Yes, treatment-related

Yes, non-treatment-related
Grade 2-4 hypertension $(n=22)$,

P-value no. $(\%)$

17 (77.3)

0.351

5 (22.7)

20 (90.9)

0.587

$2(9.1)$

$10 \quad 45.5)$

0.147

$12 \quad(54.5)$

$18 \quad(81.8)$

0.241

$6 \quad(27.3)$

$17 \quad(77.3)$

1.000

5 (22.7)

9 (40.9)

1.000

$12(54.5)$

1 (4.5)

$5 \quad(22.7)$

17 (77.3)

1.000

$9 \quad(62.3)$

0.256

5 (35.7)

7 (31.8)

0.382

$15 \quad(68.2)$

$21 \quad(95.5)$

1.000

1 (4.5)

$16 \quad(72.7)$

0.267

6 (27.3)

$10 \quad(45.5)$

0.016

$12(54.5)$

$22(100.0)$

0.493

$\begin{array}{ll}0 & (0.0)\end{array}$

7 (31.8)

0.382

8 (36.4)

7 (31.8)

${ }^{a}$ Comparison of groups 0-1 versus 2-4. TEE, thromboembolic event; ECOG, Eastern Cooperative Oncology Group; ULN, upper limit of normal; CR, complete response; $\mathrm{PR}$, partial response. 
Table VI. Association between baseline circulating biomarker levels and progression-free survival and overall survival.

\begin{tabular}{|c|c|c|c|c|c|c|c|c|}
\hline Biomarker & Cut-off (pg/ml) & No. & HR PFS & $95 \% \mathrm{CI}$ & P-value & HR OS & $95 \% \mathrm{CI}$ & P-value \\
\hline \multirow[t]{2}{*}{ Angiopoietin 1} & $>7,000$ & 21 & & & & & & \\
\hline & $<7,000$ & 23 & 0.73 & $0.38-1.43$ & 0.361 & 1.32 & $0.54-3.24$ & 0.547 \\
\hline \multirow[t]{2}{*}{ Angiopoietin 2} & $>2,700$ & 22 & & & & & & \\
\hline & $<2,700$ & 23 & 1.39 & $0.72-2.70$ & 0.319 & 1.47 & $0.60-3.60$ & 0.403 \\
\hline \multirow[t]{2}{*}{ Eotaxin } & $>120$ & 23 & & & & & & \\
\hline & $<120$ & 21 & 0.94 & $0.48-1.82$ & 0.851 & 0.66 & $0.27-1.63$ & 0.378 \\
\hline \multirow[t]{2}{*}{ FGF } & $>800$ & 21 & & & & & & \\
\hline & $<800$ & 23 & 1.40 & $0.71-2.73$ & 0.332 & 1.47 & $0.60-3.61$ & 0.403 \\
\hline \multirow[t]{2}{*}{ Follistatin } & $>800$ & 20 & & & & & & \\
\hline & $<800$ & 24 & 0.90 & $0.46-1.77$ & 0.778 & 0.68 & $0.27-1.68$ & 0.392 \\
\hline \multirow{2}{*}{ G-CSF } & $>250$ & 22 & & & & & & \\
\hline & $<250$ & 22 & 1.20 & $0.62-2.33$ & 0.584 & 0.99 & $0.40-2.44$ & 0.991 \\
\hline \multirow[t]{2}{*}{ HER 1 (EGFR) } & $>28,000$ & 22 & & & & & & \\
\hline & $<28,000$ & 22 & 0.76 & $0.39-1.49$ & 0.427 & 0.96 & $0.38-2.37$ & 0.925 \\
\hline \multirow[t]{2}{*}{ HER 2} & $>7,300$ & 22 & & & & & & \\
\hline & $<7,300$ & 22 & 0.47 & $0.23-0.94$ & 0.019 & 0.53 & $0.21-1.36$ & 0.195 \\
\hline \multirow[t]{2}{*}{ HGF } & $>1,700$ & 22 & & & & & & \\
\hline & $<1,700$ & 22 & 0.64 & $0.33-1.26$ & 0.192 & 0.42 & $0.17-1.07$ & 0.074 \\
\hline \multirow[t]{2}{*}{ ICAM1 (CD54) } & $>115,000$ & 23 & & & & & & \\
\hline & $<115,000$ & 22 & 1.60 & $0.82-3.11$ & 0.159 & 0.78 & $0.31-1.91$ & 0.579 \\
\hline \multirow[t]{2}{*}{ IL6R $\alpha$} & $>24,000$ & 22 & & & & & & \\
\hline & $<24,000$ & 21 & 0.50 & $0.25-0.98$ & 0.034 & 0.53 & $0.21-1.34$ & 0.194 \\
\hline \multirow[t]{2}{*}{ IL8 } & $>30$ & 21 & & & & & & \\
\hline & $<30$ & 23 & 0.88 & $0.45-1.72$ & 0.710 & 1.77 & $0.72-4.34$ & 0.220 \\
\hline \multirow[t]{2}{*}{ Leptin } & $>5,900$ & 22 & & & & & & \\
\hline & $<5,900$ & 22 & 0.98 & $0.50-1.89$ & 0.950 & 1.06 & $0.43-2.62$ & 0.890 \\
\hline \multirow[t]{2}{*}{ MIF } & $>6,000$ & 22 & & & & & & \\
\hline & $<6,000$ & 22 & 0.71 & $0.36-1.39$ & 0.289 & 0.58 & $0.23-1.44$ & 0.233 \\
\hline \multirow[t]{2}{*}{ NRP1 } & $>500,000$ & 22 & & & & & & \\
\hline & $<500,000$ & 21 & 1.02 & $0.52-2.00$ & 0.953 & 0.59 & $0.23-1.48$ & 0.264 \\
\hline \multirow[t]{2}{*}{ Osteopontin } & $>145,000$ & 20 & & & & & & \\
\hline & $<145,000$ & 23 & 0.66 & $0.33-1.32$ & 0.194 & 0.99 & $0.39-2.49$ & 0.977 \\
\hline \multirow[t]{2}{*}{ PDGF } & $>1,000$ & 21 & & & & & & \\
\hline & $<1,000$ & 22 & 0.66 & $0.33-1.31$ & 0.221 & 0.57 & $0.22-1.43$ & 0.233 \\
\hline \multirow[t]{2}{*}{ PECAM1 } & $>7,300$ & 21 & & & & & & \\
\hline & $<7,300$ & 23 & 0.63 & $0.32-1.22$ & 0.160 & 0.41 & $0.16-1.00$ & 0.058 \\
\hline \multirow[t]{2}{*}{ PlGF } & $>20$ & 4 & & & & & & \\
\hline & $<20$ & 40 & 0.32 & $0.06-1.74$ & 0.021 & 0.31 & $0.04-2.23$ & 0.044 \\
\hline \multirow[t]{2}{*}{ Prolactin } & $>7,000$ & 23 & & & & & & \\
\hline & $<7,000$ & 20 & 0.93 & $0.48-1.83$ & 0.840 & 0.62 & $0.25-1.57$ & 0.322 \\
\hline SCF & $>400$ & 22 & & & & & & \\
\hline & $<400$ & 21 & 1.12 & $0.57-2.22$ & 0.740 & 0.82 & $0.32-2.07$ & 0.668 \\
\hline SDF1 $\alpha$ & $>135$ & 20 & & & & & & \\
\hline (CXCL12) & $<135$ & 24 & 0.84 & $0.43-1.63$ & 0.598 & 0.80 & $0.32-1.97$ & 0.625 \\
\hline SPD & $>9,600$ & 22 & & & & & & \\
\hline & $<9,600$ & 22 & 1.69 & $0.86-3.31$ & 0.106 & 2.10 & $0.85-5.19$ & 0.106 \\
\hline Tenascin C & $>10,000$ & 22 & & & & & & \\
\hline & $<10,000$ & 22 & 0.62 & $0.32-1.23$ & 0.152 & 0.40 & $0.16-1.00$ & 0.047 \\
\hline
\end{tabular}


Table VI. Continued.

\begin{tabular}{|c|c|c|c|c|c|c|c|c|}
\hline Biomarker & Cut-off (pg/ml) & No. & HR PFS & $95 \% \mathrm{CI}$ & P-value & HR OS & $95 \% \mathrm{CI}$ & P-value \\
\hline \multirow[t]{2}{*}{ TIE2 } & $>20,000$ & 16 & & & & & & \\
\hline & $<20,000$ & 27 & 0.70 & $0.34-1.46$ & 0.299 & 0.49 & $0.19-1.28$ & 0.129 \\
\hline \multirow[t]{2}{*}{ VCAM1 } & $>1,300,000$ & 23 & & & & & & \\
\hline & $<1,300,000$ & 21 & 0.92 & $0.48-1.79$ & 0.812 & 0.70 & $0.28-1.72$ & 0.438 \\
\hline \multirow[t]{2}{*}{ VEGF-A } & $>0$ & 17 & & & & & & \\
\hline & 0 & 27 & 0.66 & $0.32-1.39$ & 0.232 & 0.67 & $0.26-1.74$ & 0.379 \\
\hline \multirow[t]{2}{*}{ VEGF-C } & $>800$ & 18 & & & & & & \\
\hline & $<800$ & 26 & 1.72 & $0.89-3.35$ & 0.098 & 1.41 & $0.57-3.47$ & 0.469 \\
\hline \multirow[t]{2}{*}{ VEGFR1 } & $>1,300$ & 22 & & & & & & \\
\hline & $<1,300$ & 21 & 0.98 & $0.50-1.92$ & 0.957 & 1.06 & $0.42-2.67$ & 0.901 \\
\hline \multirow[t]{2}{*}{ VEGFR2 } & $>7,000$ & 22 & & & & & & \\
\hline & $<7,000$ & 21 & 0.96 & $0.49-1.89$ & 0.905 & 1.10 & $0.43-2.80$ & 0.840 \\
\hline \multirow[t]{2}{*}{ VEGFR3 } & $>2,250$ & 21 & & & & & & \\
\hline & $<2,250$ & 23 & 1.74 & $0.89-3.40$ & 0.093 & 1.52 & $0.62-3.74$ & 0.361 \\
\hline
\end{tabular}

FGF, fibroblast growth factor; G-CSF, granulocyte colony-stimulating factor; HER/EGFR, epidermal growth factor receptor; HER2, human epidermal growth factor receptor 2; HGF, hepatocyte growth factor; ICAM1, intercellular adhesion molecule 1 (also known as CD54); IL8, interleukin 8; MIF, migration inhibitory factor; NRP1, neuropilin-1; PDGF, platelet-derived growth factor; PIGF, placental growth factor; SCF, stem cell factor; SDF1 $\alpha$, stromal cell-derived factor $1 \alpha$; SPD, spindle-defective protein; VCAM1, vascular cell adhesion molecule 1; VEGF, vascular endothelial growth factor.
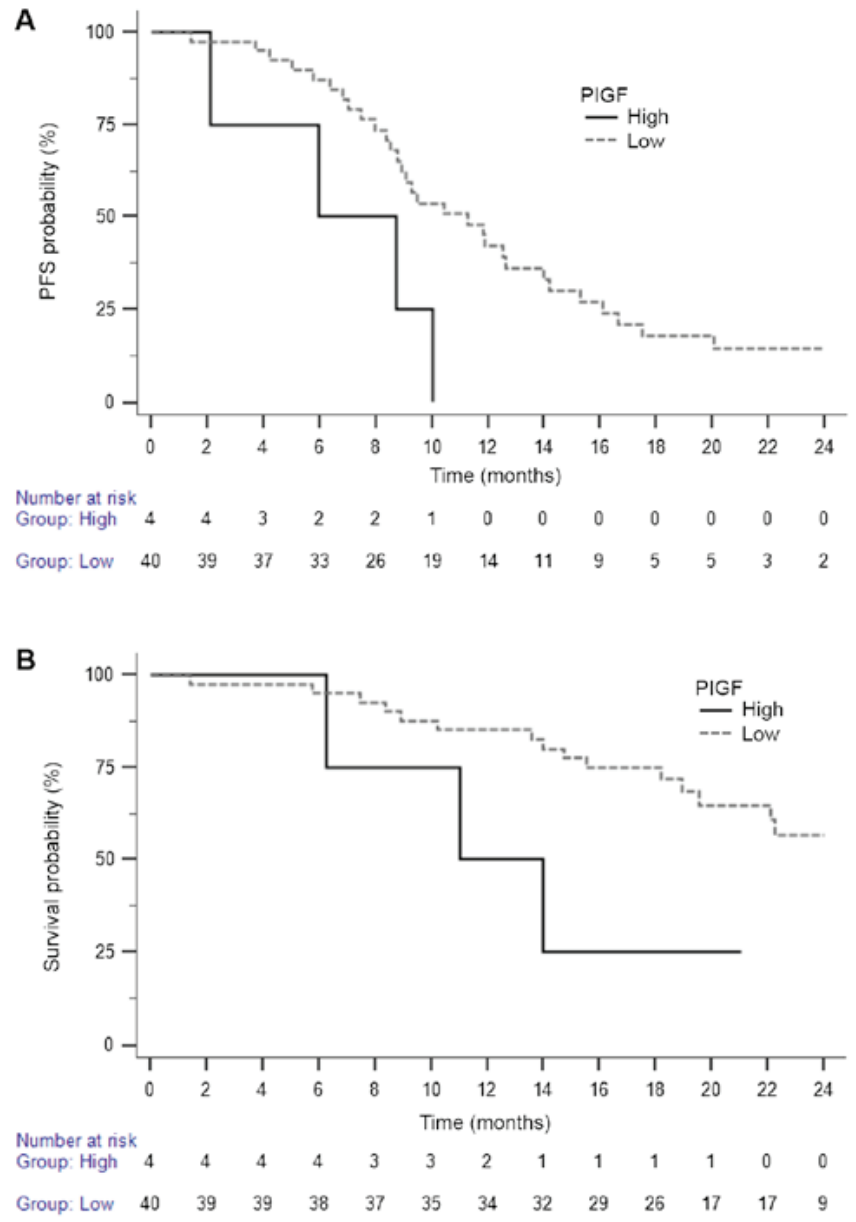

Figure 2. (A) Progression-free survival (PFS); and (B) overall survival curves according to baseline placental growth factor (PIGF) levels.
(Table VI and Fig. 2). There was a trend for an association between the high on-treatment PIGF level and the occurrence of grade 2-4 diarrhea $(\mathrm{P}=0.086)$, but not with hypertension $(\mathrm{P}=0.256)$.

\section{Discussion}

VELVET was the first phase II study evaluating aflibercept with an oxaliplatin stop-and-go strategy in patients with previously untreated and unresectable mCRC. The targeted 85\% 6-month PFS rate was not reached in the ITT population: The absolute rate and the Kaplan-Meier estimates of 6-month PFS were 67 and $79 \%$, respectively. The ORR was 59\% and median PFS and OS were 9.3 months and 22.2 months, respectively. The maintenance rate $(79 \%)$ was higher than in previous oxaliplatin stop-and-go studies $(10,21,27)$.

In the OPTIMOX1 and OPTIMOX2 studies $(10,21)$ a similar oxaliplatin stop-and-go strategy without anti-angiogenic agent led to a response rate of $59.2 \%$ and median PFS $<9$ months. In the AFFIRM randomized phase II study (22), 236 patients with unresectable $\mathrm{mCRC}$ were randomized between first-line FOLFOX $(n=117)$ and FOLFOX-aflibercept $(n=119)$ until progression. That study was conducted in Europe, Asia and Australia, regions with different clinical guidelines for the treatment of mCRC. The 1-year PFS rate (primary endpoint) was similar in both groups (21.2 versus $25.8 \%$ ). There was no significant improvement in efficacy endpoints with the addition of aflibercept to chemotherapy (ORR, 45.9 versus $49.1 \%$; median PFS, 8.8 versus 8.5 months; and median OS, 22.3 versus 19.5 months) and in salvage surgery rate (5.1 versus 5.0\%). In the NO16966 study (4) the addition of 


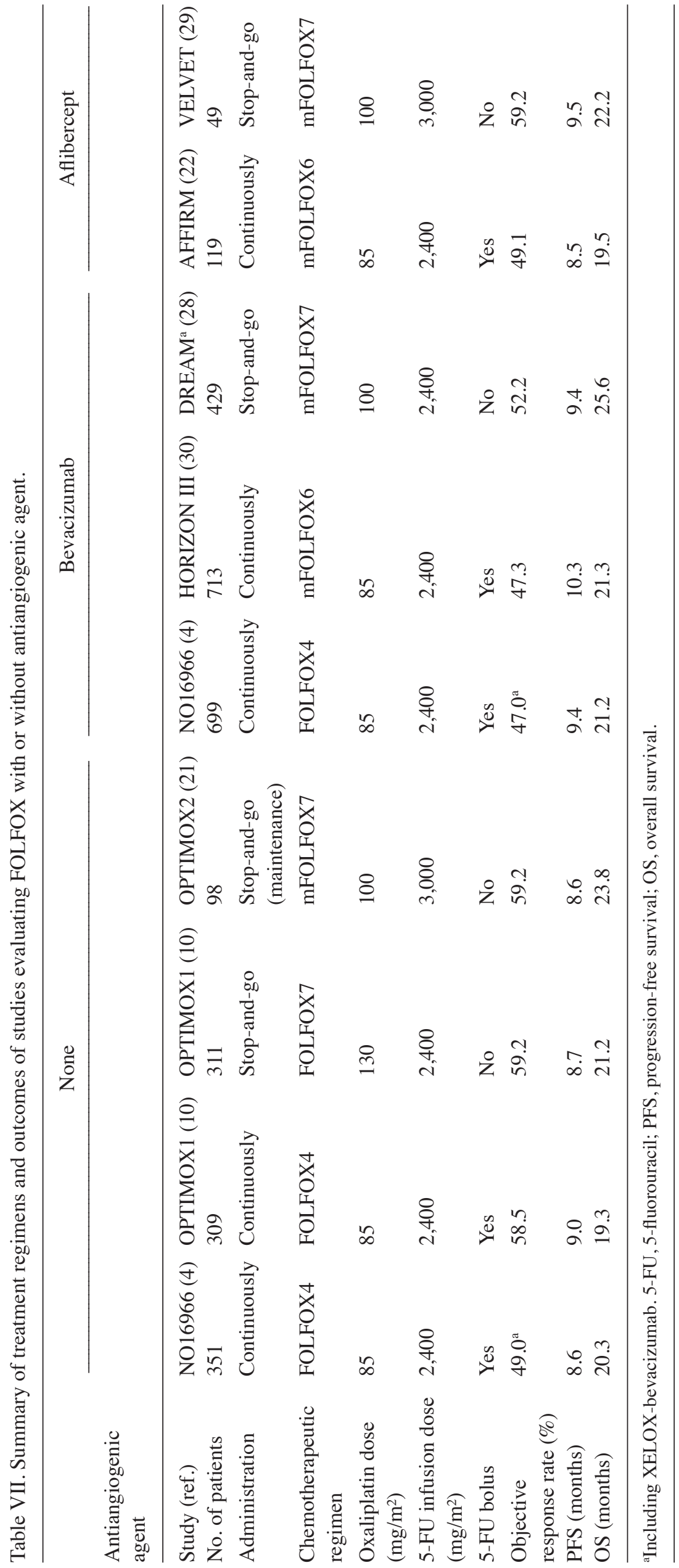


bevacizumab to an oxaliplatin-based chemotherapy (FOLFOX or XELOX) led to an improvement in PFS (primary endpoint) from 8.0 to 9.5 months (HR, 0.83; $\mathrm{P}=0.002$ ). This benefit was greater when patients were censored at the time of drug discontinuation ('on-treatment PFS'; HR, 0.63). The median PFS in patients who received FOLFOX4-bevacizumab was 9.4 months. The ORR was similar whether patients received chemotherapy with $(47 \%)$ or without $(49 \%)$ bevacizumab. The oxaliplatin-based stop-and-go strategy with bevacizumab was previously evaluated in several randomized phase III trials (11,23-26). Among 700 patients enrolled in the DREAM study (27), $429(61.3 \%)$ received an induction therapy with modified FOLFOX7 plus bevacizumab, using the same dose of oxaliplatin $\left(100 \mathrm{mg} / \mathrm{m}^{2}\right)$ than in the present study, although a lower dose of 5-FU infusion. In those patients, the ORR was $52.2 \%$ and the median PFS was 9.4 months (28). Thus, the addition of aflibercept to an oxaliplatin stop-and-go strategy in patients with unresectable mCRC seems to increase PFS to the same degree as bevacizumab (from $<9$ to 9.5 months) and to slightly increase the tumor ORR (Table VII). This effect may also be associated with higher doses of 5-FU infusion.

In the present study, the frequency of severe (grade 3 or 4 ) hypertension $(23 \%)$ was similar to that reported in the VELOUR trial (19\%) (29), although lower than described in the AFFIRM study (36\%) (22). When adding bevacizumab to an oxaliplatin-based chemotherapy in patients with advanced mCRC, the incidence of grade 3-4 hypertension ranges between 4 and $6 \%(4,30-32)$. In this study, this adverse event occurred mainly during induction therapy, and was reversed in most cases before maintenance therapy. Of note, a high systolic blood pressure $(\geq 140 \mathrm{mmHg})$ at study entry was associated with shorter PFS and a higher frequency of treatment induced grade 2-4 hypertension.

The exposure to aflibercept with FOLFOX was associated with an increase in PIGF levels after the first infusion. When trapping circulating PlGF, aflibercept inhibits the binding to VEGF receptors 1 and 2, thus increasing the circulating PlGF level.

Despite the statistically negative result of this study, but given the high response rate, OPTIMOX-aflibercept may be an active first-line treatment strategy in patients with previously untreated and unresectable $\mathrm{mCRC}$, providing strict monitoring of blood pressure and immediate management of hypertension during therapy. Further trials evaluating this combination should provide early safety analysis.

\section{Acknowledgements}

The authors would like to thank the patients who participated in this study, their families, the coordinating staff in GERCOR (particularly Ms. Attia Malika) and all the following investigators: Dr Artru Pascal (Hôpital Privé Jean Mermoz, Lyon, France), Dr Savinelli Francesco, and Dr Sverdlin Robert (Hôpital Privé Saint-Joseph, Paris, France). The authors also acknowledge the assistance of Benetkiewicz Magdalena in manuscript preparation. Her work was funded by GERCOR.

\section{Funding}

This study was supported by Sanofi. Sanofi did not have any role in the study design, collection, analysis and interpretation of the data, the writing of the manuscript and the decision to submit the study for publication.

\section{Availability of data and materials}

The datasets used and analyzed during the current study are available from the corresponding author on request.

\section{Authors' contributions}

$\mathrm{BC}$ and $\mathrm{ADG}$ were responsible for the conception and design of the study. CT, BC, TA, WS, and ADG recruited the patients. BC, JBB, TA, DA, JDe, GD, CLe, CLo, CT, VL, JDa, GL, MLG, OD, NBH, AM, AKL, and ATR collected the data. BC, FB, and AdG analyzed the data. CT and AdG interpreted the data. BC and AdG wrote the manuscript. All authors have edited, read and approved the final manuscript.

\section{Ethics approval and consent to participate}

The study was carried out in accordance with the declaration of Helsinki and Good Clinical Practice guidelines. All patients provided written informed consent. This study was approved by the Ethics Committee of our institution (CPP Ile de France VI Groupe Hospitalier Pitié Salpêtrière PARIS).

\section{Patient consent for publication}

Not applicable.

\section{Competing interests}

BC reported personal fees from Roche Pharma AG, Amgen, Sanofi and Menarini. JBB reported personal fees from Amgen, Bayer, Celgène, Merck Serono, Roche, Sanofi and Roche. TA reported personal fees from BMS, Roche, MSD Oncology, Sanofi, Novartis, Servier, Amgen, Lilly, Xbiotec, Mundipharma and Yacult. All remaining authors have declared no competing interests.

\section{References}

1. American Cancer Society: Cancer facts and figures 2015 http://www.cancer.org/research/cancerfactsstatistics/cancerfactsfigures2015/index. Accessed, May 14, 2018.

2. Chibaudel B, Tournigand C, Bonnetain F, Richa H, M, André T and de Gramont A: Therapeutic strategy in unresectable metastatic colorectal cancer: An updated review. Ther Adv Med Oncol 7: 153-169, 2015.

3. Hurwitz H,Fehrenbacher L, Novotny W, Cartwright T, Hainsworth J, Heim W, Berlin J, Baron A, Griffing S, Holmgren E, et al: Bevacizumab plus irinotecan, fluorouracil, and leucovorin for metastatic colorectal cancer. N Engl J Med 350: 2335-2342, 2004.

4. Saltz LB, Clarke S, Díaz-Rubio E, Scheithauer W, Figer A, Wong R, Koski S, Lichinitser M, Yang TS, Rivera F, et al: Bevacizumab in combination with oxaliplatin-based chemotherapy as first-line therapy in metastatic colorectal cancer: A randomized phase III study. J Clin Oncol 26: 2013-2019, 2008.

5. Loupakis F, Cremolini C, Masi G, Lonardi S, Zagonel V, Salvatore L, Cortesi E, Tomasello G, Ronzoni M, Spadi R, et al: Initial therapy with FOLFOXIRI and bevacizumab for metastatic colorectal cancer. N Engl J Med 371: 1609-1618, 2014.

6. Van Cutsem E, Köhne CH, Hitre E, Zaluski J, Chang Chien CR, Makhson A, D'Haens G, Pintér T, Lim R, Bodoky G, et al: Cetuximab and chemotherapy as initial treatment for metastatic colorectal cancer. N Engl J Med 360: 1408-1417, 2009. 
7. Douillard JY, Siena S, Cassidy J, Tabernero J, Burkes R, Barugel M, Humblet Y, Bodoky G, Cunningham D, Jassem J, et al: Randomized, phase III trial of panitumumab with infusional fluorouracil, leucovorin, and oxaliplatin (FOLFOX4) versus FOLFOX4 alone as first-line treatment in patients with previously untreated metastatic colorectal cancer: The PRIME study. J Clin Oncol 28: 4697-4705, 2010.

8. Douillard JY, Oliner KS, Siena S, Tabernero J, Burkes R, Barugel M, Humblet Y, Bodoky G, Cunningham D, Jassem J, et al: Panitumumab-FOLFOX4 treatment and RAS mutations in colorectal cancer. N Engl J Med 369: 1023-1034, 2013.

9. de Gramont A, Figer A, Seymour M, Homerin M, Hmissi A, Cassidy J, Boni C, Cortes-Funes H, Cervantes A, Freyer G, et al: Leucovorin and fluorouracil with or without oxaliplatin as first-line treatment in advanced colorectal cancer. J Clin Oncol 18: 2938-2947, 2000.

10. Tournigand C, Cervantes A, Figer A, Lledo G, Flesch M, Buyse M, Mineur L, Carola E, Etienne PL, Rivera F, et al: OPTIMOX1: A randomized study of FOLFOX4 or FOLFOX7 with oxaliplatin in a stop-and-Go fashion in advanced colorectal cancer--a GERCOR study. J Clin Oncol 24: 394-400, 2006.

11. Simkens LH, van Tinteren H, May A, ten Tije AJ, Creemers GJ, Loosveld OJ, de Jongh FE, Erdkamp FL, Erjavec Z, van der Torren AM, et al: Maintenance treatment with capecitabine and bevacizumab in metastatic colorectal cancer (CAIRO3): A phase 3 randomised controlled trial of the Dutch Colorectal Cancer Group. Lancet 385: 1843-1852, 2015.

12. Van Cutsem E, Tabernero J, Lakomy R, Prenen H, Prausová J, Macarulla T, Ruff P, van Hazel GA, Moiseyenko V, Ferry D, et al: Addition of aflibercept to fluorouracil, leucovorin, and irinotecan improves survival in a phase III randomized trial in patients with metastatic colorectal cancer previously treated with an oxaliplatin-based regimen. J Clin Oncol 30: 3499-3506, 2012.

13. Chibaudel B, Bonnetain F, Shi Q, Buyse M, Tournigand C, Sargent DJ, Allegra CJ, Goldberg RM and de Gramont A: Alternative end points to evaluate a therapeutic strategy in advanced colorectal cancer: Evaluation of progression-free survival, duration of disease control, and time to failure of strategy--an Aide et Recherche en Cancerologie Digestive Group Study. J Clin Oncol 29: 4199-4204, 2011

14. Eisenhauer EA, Therasse P, Bogaerts J, Schwartz LH, Sargent D, Ford R, Dancey J, Arbuck S, Gwyther S, Mooney M, et al: New response evaluation criteria in solid tumours: Revised RECIST guideline (version 1.1). Eur J Cancer 45: 228-247, 2009.

15. National Canceer Institute: Common terminology criteria for adverse events 9CTCAE), v4.03. http://evs.nci.nih. gov/ftp1/CTCAE/CTCAE_4.03_2010-06-14_QuickReference_ 5x7.pdf. Accessed, May 14, 2018

16. EORTC Quality of Life Group: EORTC QLQ-C30 (version 3) Brussels: EORTC Quality of Life Group; 1995.http://groups.eortc. be/qol/sites/default/files/img/slider/specimen_qlq-c30_english.pdf. Accessed, May 14, 2018.

17. Chibaudel B, Bonnetain F, Tournigand C, Bengrine-Lefevre L, Teixeira L, Artru P, Desramé J, Larsen AK, André T, Louvet C, et al: Simplified prognostic model in patients with oxaliplatin-based or irinotecan-based first-line chemotherapy for metastatic colorectal cancer: A GERCOR study. Oncologist 16: 1228-1238, 2011

18. Simon R: Optimal two-stage designs for phase II clinical trials. Control Clin Trials 10: 1-10, 1989

19. Schemper M and Smith TL: A note on quantifying follow-up in studies of failure time. Control Clin Trials 17: 343-346, 1996.

20. Kaplan EL and Meier P: Non parametric estimation from incomplete observations. J Am Stat Assoc 53: 457-481, 1958.

21. Chibaudel B, Maindrault-Goebel F, Lledo G, Mineur L, André T, Bennamoun M, Mabro M, Artru P, Carola E, Flesch M, et al: Can chemotherapy be discontinued in unresectable metastatic colorectal cancer? The GERCOR OPTIMOX2 Study. J Clin Oncol 27: 5727-5733, 2009.

22. Folprecht G, Pericay C, Saunders MP, Thomas A, Lopez Lopez R, Roh JK, Chistyakov V, Höhler T, Kim JS, Hofheinz RD, et al: Oxaliplatin and 5-FU/folinic acid (modified FOLFOX6) with or without aflibercept in first-line treatment of patients with metastatic colorectal cancer: The AFFIRM study. Ann Oncol 27: $1273-1279,2016$.
23. Tournigand C, Lledo G, Delord J, André T, Maindrault-Goebel F, Louvet C, Scheithauer W and de Gramont A: Modified Folfox7/bevacizumab or modified Xelox/bevacizumab with or without erlotinib in first-line metastatic colorectal cancer: Results of the feasability phase of the DREAM-OPTIMOX3 study (GERCOR). J Clin Oncol 25 (Suppl. 18): 4097, 2007.

24. Koopman M, Simkens Lieke HJ, Ten Tije AJ, Creemers GJ, Loosveld OJ, De Jongh FE, Erdkamp F, Erjavec Z, van der Torren AME, et al: Maintenance treatment with capecitabine and Bevacizumab versus observation after induction treatment with chemotherapy and Bevacizumab in metastatic colorectal cancer (mCRC): The phase Iii Cairo3 Study of the Dutch Colorectal Cancer Group (DCCG). J Clin Oncol 31 (Suppl): 3502, 2013.

25. Arnold D, Graeven U,Lerchenmuller C, Killing B, Depenbusch R, Steffens C, Salah-Eddin Al-Batran S-E, Lange T, Dietrich G, Jan Stoehlmacher J, et al: Maintenance strategy with fluoropyrimidines (FP) plus Bevacizumab (Bev), Bev alone, or no treatment, following a standard combination of FP, oxaliplatin $(\mathrm{Ox})$, and $\mathrm{Bev}$ as first-line treatment for patients with metastatic colorectal cancer (mCRC): A phase III non-inferiority trial (AIO KRK 0207). J Clin Oncol 32 (Suppl. 5): 3503, 2014.

26. Koeberle D, Betticher DC, von Moos R, Dietrich D, Brauchli P, Baertschi D, Matter K, Winterhalder R, Borner M, Anchisi S, et al: Bevacizumab continuation versus no continuation after first-line chemotherapy plus bevacizumab in patients with metastatic colorectal cancer: A randomized phase III non-inferiority trial (SAKK 41/06). Ann Oncol 26: 709-714, 2015.

27. Tournigand C, Chibaudel B, Samson B, Scheithauer W, Vernerey D, Mésange P, Lledo G, Viret F, Ramée JF, Tubiana-Mathieu N, et al: Bevacizumab with or without erlotinib as maintenance therapy in patients with metastatic colorectal cancer (GERCOR DREAM; OPTIMOX3): A randomised, open-label, phase 3 trial. Lancet Oncol 16: 1493-1505, 2015.

28. Chibaudel B, Tournigand C, Samson B, Scheithauer W, Mesange P, Lledo G, Viret F, JRamée JF, Tubiana-Mathieu N, Dauba J, et al: Bevacizumab-erlotinib as maintenance therapy in metastatic colorectal cancer. Final results of the GERCOR DREAM study. Ann Oncol 25 (Suppl. 4): iv167-iv209, 2014

29. Tabernero J, Van Cutsem E, Lakomý R, Prausová J, Ruff P, van Hazel GA, Moiseyenko VM, Ferry DR, McKendrick JJ, Soussan-Lazard K, et al: Aflibercept versus placebo in combination with fluorouracil, leucovorin and irinotecan in the treatment of previously treated metastatic colorectal cancer: Prespecified subgroup analyses from the VELOUR trial. Eur J Cancer 50: 320-331, 2014.

30. Schmoll HJ, Cunningham D, Sobrero A, Karapetis CS, Rougier P, Koski SL, Kocakova I, Bondarenko I, Bodoky G, Mainwaring P, et al: Cediranib with mFOLFOX6 versus bevacizumab with mFOLFOX6 as first-line treatment for patients with advanced colorectal cancer: A double-blind, randomized phase III study (HORIZON III). J Clin Oncol 30: 3588-3595, 2012.

31. Díaz-Rubio E, Gómez-España A, Massutí B, Sastre J, Abad A, Valladares M, Rivera F, Safont MJ, Martínez de Prado P, Gallén M, et al; Spanish Cooperative Group for the Treatment of Digestive Tumors: First-line XELOX plus bevacizumab followed by XELOX plus bevacizumab or single-agent bevacizumab as maintenance therapy in patients with metastatic colorectal cancer: The phase III MACRO TTD study. Oncologist 17: 15-25, 2012.

32. Giantonio BJ, Catalano PJ, Meropol NJ, O'Dwyer PJ, Mitchell EP, Alberts SR, Schwartz MA and Benson AB III; Eastern Cooperative Oncology Group Study E3200: Bevacizumab in combination with oxaliplatin, fluorouracil, and leucovorin (FOLFOX4) for previously treated metastatic colorectal cancer: Results from the Eastern Cooperative Oncology Group Study E3200. J Clin Oncol 25: 1539-1544, 2007. 